\title{
O uso de praias arenosas com diferentes concentrações humanas por espécies de aves limícolas (Charadriidae e Scolopacidae) neárticas no sudeste do Brasil
}

\author{
César Cestari ${ }^{1,2}$ \\ ${ }^{1}$ Departamento de Zoologia, Instituto de Biociências, \\ Universidade Estadual Paulista Júlio de Mesquita Filho - UNESP, \\ Av. 24-A, 1515, CP 199, CEP 13506-900, Rio Claro, SP, Brasil \\ ${ }^{2}$ Autor para correspondência: César Cestari,e-mail: cesar_cestari@yahoo.com.br
}

CESTARI, C. The use of sandy beaches with different concentration of humans by Nearctic shorebirds (Charadriidae and Scolopacidae) in southeastern Brazil. Biota Neotrop. 8(4): http://www.biotaneotropica. org.br/v8n4/en/abstract?article+bn01308042008

\begin{abstract}
Migratory birds may recognize humans and domestic animals as potential predators. Thus, their abundance and behavior in flock formation may change in areas with high human concentration. This study compared the abundance of Nearctic migratory birds, the frequency of their flocks and the average number of birds per flock at high and low human concentration areas in a coastal region in southeastern Brazil. Birds, humans and domestic dogs were counted monthly between November 2006 and April 2007. Six bird species (Ruddy Turnstone Arenaria interpres, Sanderling Calidris alba, Red Knot Calidris canutus, Semipalmated Sandpiper Calidris pusilla, Semipalmated Plover Charadrius semipalmatus, and American Golden-Plover Pluvialis dominica) were recorded. Only Red Knot were restricted to the low human concentration area. The average number of humans and domestic dogs differed between areas with different levels of disturbance, whereas no difference on the average number of birds was detected. There were no correlation between number of humans and birds, nor between domestic dogs and birds. Additionally, the frequency of flocks and average number of birds per flock did not vary significantly between the areas. These results highlighted the sensibility of Red Knot in the high human concentration area as well as the need to find out, in future investigations, the maximum concentration of people and domestic dogs that birds can tolerate in human occupied areas to employ conservation efforts in preserving Nearctic shorebirds.
\end{abstract}

Keywords: human disturbance, sandy beaches, Scolopacidae, shorebirds.

CESTARI, C. O uso de praias arenosas com diferentes concentrações humanas por espécies de aves limícolas (Charadriidae e Scolopacidae) neárticas no sudeste do Brasil. Biota Neotrop. 8(4): http://www.biotaneotropica. org.br/v8n4/pt/abstract?article+bn01308042008

Resumo: As aves migratórias podem reconhecer humanos e animais domésticos como possíveis predadores, alterando seus padrões de abundância e comportamento de formação de bandos. O objetivo do presente estudo foi comparar a abundância de aves migratórias neárticas, a freqüência de bandos e o número médio de aves por bando em áreas com alta e baixa concentração humana em uma região costeira de praia arenosa no sudeste do Brasil. As aves, pessoas e cães foram contados mensalmente entre novembro de 2006 a abril de 2007. Foram registradas seis espécies de aves (Arenaria interpres, Calidris alba, Calidris canutus, Calidris pusilla, Charadrius semipalmatus, Pluvialis dominica) nas duas áreas, no entanto somente $C$. canutus foi registrado exclusivamente na área com baixa concentração humana. Houve diferença significativa no número médio de pessoas e cães entre as áreas, mas não no número médio de aves. Não houve correlação entre o número de humanos e aves, e entre cães e aves. Adicionalmente, não houve diferença significativa na frequiência de bandos e número de indivíduos por bando entre as áreas. Os resultados deste estudo destacaram a sensibilidade de C. canutus na área com alta concentração humana e a necessidade de futuras investigações que determinem os limites máximos de concentração de pessoas e cães domésticos que as aves migratórias neárticas podem tolerar para a tomada de ações de proteção em áreas costeiras com ocupação humana.

Palavras-chave: aves costeiras, perturbação humana, praias arenosas, Scolopacidae. 


\section{Introdução}

O avanço da urbanização em áreas costeiras pode interferir negativamente na sobrevivência, comportamentos, presença e abundância de aves migratórias (Burger 1993, Hubbard \& Dugan 2003, Thomas et al. 2003, Burton et al. 2006). Comparando com outras espécies de aves costeiras, as aves migratórias podem ser altamente vulneráveis às atividades recreativas humanas (Burger 1981). Alguns dos principais impactos negativos causados pela intensa atividade humana em áreas costeiras são o afastamento e a diminuição da freqüência de forrageamento de aves migratórias (Burger \& Gochfeld 1991, Burger et al. 2004).

Algumas espécies de aves migratórias limícolas, conhecidas popularmente como batuíras e maçaricos (Charadriidae e Scolopacidae), reproduzem-se na América do Norte durante o verão boreal e, no inverno, deslocam-se para a região costeira da América do Sul, em busca de locais de pouso e alimentação (Sick 1997, Larrazábal et al. 2002). O acúmulo de reservas de gordura durante o período de invernagem é determinante para o retorno dessas aves para as áreas de reprodução e, muitas vezes, a perturbação provocada por atividades humanas influenciam em um maior gasto de energia na procura de locais menos impactados (Vooren \& Chiaradia 1990). Estudos demonstram o declínio recente de algumas populações de maçaricos e batuíras que visitam sazonalmente o Brasil, principalmente devido à perda de hábitats e diminuição de alimentos por consequiência da ocupação e atividades humanas (Morrison et al. 1989, Morrison et al. 2004). Calidris canutus rufa constitui um exemplo emblemático, com estimativas de declínio populacional de aproximadamente $3 \%$ ao ano desde a década de 90 (Morrison 2004).

O monitoramento de aves migratórias é importante para avaliar a qualidade dos locais que habitam e para tomada de medidas preventivas quanto ao declínio de suas populações (Hubbard \& Dugan 2003, Burger et al. 2004). Vários exemplos de medidas eficazes para proteção de aves migratórias contra ameaças humanas são citados por Burger et al. (2004), tais como: a instalação de placas com informações educativas, fiscalização, restrição ao acesso em áreas de uso freqüente das aves e construção de plataformas para observação das aves. No Brasil, existem estudos de monitoramento e pesquisas acerca da distribuição geográfica e padrões sazonais de ocorrência de aves migratórias realizados principalmente nas regiões norte, sul e nordeste (Morrison et al. 1989, Vooren \& Chiaradia 1990, Rodrigues 2000, Azevedo-Júnior et al. 2001, Larrazábal et al. 2002), sendo as duas primeiras regiões de grande importância para aves neárticas (Morrison et al. 1989). No entanto, estudos investigando diretamente a influência humana na ocorrência e atividades comportamentais das aves migratórias neárticas em território brasileiro ainda são escassos em comparação com o número de pesquisas realizadas em regiões costeiras da América do Norte (Burger \& Gochfeld 1991, Burger et al. 2004, Yasué 2005, 2006, Thomas et al. 2003).

O sudeste do Brasil caracteriza-se pelo mais alto índice demográfico humano do país, concentrando aproximadamente $42 \%$ do total da população (IBGE 2000). Parte dessa população se estabelece temporariamente ou definitivamente em cidades costeiras, utilizandoas principalmente como locais de lazer e trabalho. Tal ocupação pode influenciar a presença, abundância e comportamento das aves migratórias neárticas. Considerando esses fatos, o presente trabalho teve como objetivo estudar aves migratórias neárticas em uma região com áreas de praias arenosas caracterizadas, respectivamente, por alta e baixa concentração humana no sudeste do Brasil. Dessa forma, foi investigado especificamente se existe diferença significativa no número de espécies, número de indivíduos, frequiência de bandos e número de indivíduos por bando de batuíras e maçaricos neárticos em duas áreas com diferentes concentrações de humanos. Essa questão baseou-se (1) na hipótese de que algumas espécies de aves migratórias identificam os humanos e animais domésticos como possíveis predadores (Frid \& Dill 2002) e (2) no fato de que a formação de bandos reduz o risco de predação de seus integrantes pela ação de indivíduos sentinelas para proteção do grupo, existindo a tendência das aves despenderem menos tempo alertas conforme o aumento da quantidade de indivíduos pertencentes ao bando (Elgard 1989, Cresswell 1994, Mori et al. 2001). Assim, a primeira hipótese pode ser confirmada se os resultados indicarem uma maior abundância de aves na área com baixa concentração humana. Em caso negativo, se ocorrer presença regular das aves nas duas áreas de estudo, espera-se que exista uma maior freqüência de bandos e um número maior de indivíduos por bando de aves em áreas com maior concentração humana e elevado risco de predação, confirmando a segunda hipótese.

\section{Material e Métodos}

\section{1. Área de estudo}

O estudo foi realizado em uma extensão de $9 \mathrm{~km}$ de praia arenosa (entre as latitudes $24^{\circ} 13^{\prime} 28,9^{\prime \prime}$ e $24^{\circ} 16^{\prime} 23,4^{\prime \prime} \mathrm{S}$ e longitudes $46^{\circ} 51^{\prime} 20,2^{\prime \prime}$ e $46^{\circ} 55^{\prime} 52,1^{\prime \prime}$ W) abrangendo os municípios de Itanhaém e Peruíbe, costa sul do estado de São Paulo. A região de estudo compreendeu duas áreas com diferentes concentrações de humanos: 1) área de alta concentração, com $4,5 \mathrm{~km}$ de praias contínuas paralelas a bairros periféricos de Itanhaém e, 2) área de baixa concentração, com 4,5 km de praias contínuas paralelas à vegetação de Restinga ainda não ocupada pela expansão urbana do município de Peruíbe. A segunda área de praia faz transição como um dos últimos remanescentes de vegetação nativa de Restinga entre a região sul da cidade de Santos a Peruíbe (Olmos \& Galetti 2004) (Figura 1).

Por se tratarem de faixas contínuas de areia, as duas áreas não possuem diferenças físicas e estruturais evidentes, apresentando relevo uniformemente plano, com areia compacta e úmida na maior porção onde a maré alcança e, areia seca e fofa em uma pequena extensão fora do alcance da maré (aproximadamente $10 \mathrm{~m}$ ). A coleta dos dados foi realizada em dias consecutivos visando uma mínima variação de ação da maré nas áreas. Assim, a largura média da paisagem efetiva

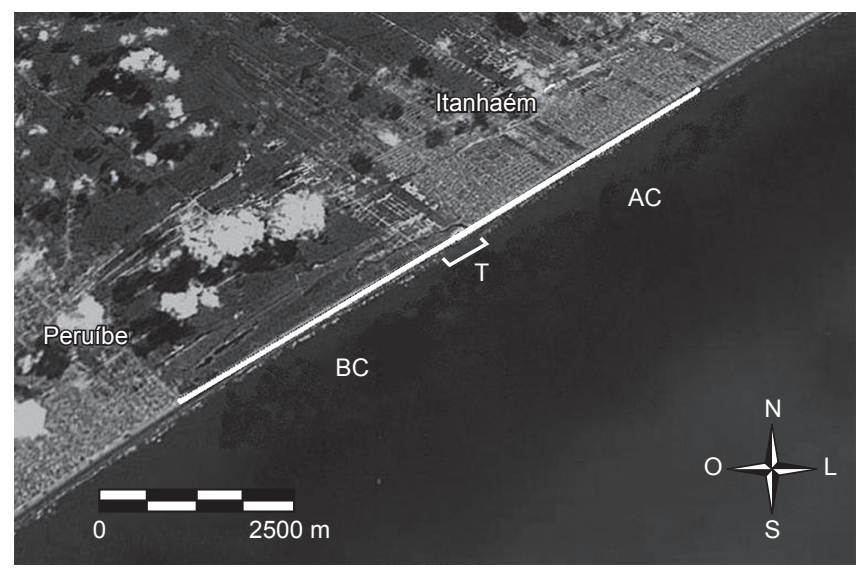

Figura 1. Áreas de estudo indicando onde foram realizados os registros das aves migratórias neárticas ( $\mathrm{BC}$ - área com baixa concentração humana; $\mathrm{AC}$ - área com alta concentração humana; $\mathrm{T}$ - Área de transição). Modificado de Google Earth 2008: www.google.com/earth.

Figure 1. Study areas where the Nearctic birds were registered (BC - Area with low human concentration; $\mathrm{AC}$ - Area with high human concentration; $\mathrm{T}$ - Transition area). Modified from Google Earth 2008: www.google.com/ earth. 
de estudo (faixa de areia exposta perpendicular ao oceano) para as duas áreas foi de $87 \pm 9 \mathrm{~m}$.

\section{Metodologia}

O estudo foi conduzido entre novembro de 2006 e abril de 2007, principais meses em que as aves costumam migrar de regiões mais setentrionais do continente americano e freqüentar a costa brasileira (Sick 1997). Durante esse período de invernagem, as aves utilizam a costa do Brasil como local de pouso e alimentação ou como área de passagem em sua rota de migração. Esse período coincide também com a principal época de férias e deslocamento de pessoas para praias da região em busca de lazer.

A coleta de dados foi realizada ao longo de uma transecção em áreas com alta e baixa concentração humana. Para isso foram utilizados binóculos $8 \times 30 \mathrm{~mm}$, gravador portátil, GPS e uma bicicleta. Cada área foi percorrida e amostrada por quatro dias consecutivos ao mês, totalizando 24 contagens por área. Nas contagens, efetuadas entre 8:00 e 10:30, foram registradas no gravador as seguintes informações: 1) número de pessoas e de cães domésticos; 2) espécies de aves e respectivas abundâncias; 3) se as aves estavam em bandos (heteroespecíficos ou monoespecíficos) ou isoladas e, 4) número de indivíduos por bando. Os indivíduos espaçados em menos de $5 \mathrm{~m}$ entre si foram considerados como pertencentes a um único bando. Esse limite máximo de espaçamento para diferenciação de bandos foi adotado por caracterizar coesão e deslocamento unidirecional dos indivíduos pertencentes a um mesmo bando quando se movimentavam em solo ou em vôo no momento em que eram afugentados por pessoas ocupando a praia (obs. pess.).

Para discriminar claramente a presença das aves nas duas áreas, foi estabelecido um trecho de transição de $1 \mathrm{~km}$ onde não foram coletados os dados descritos acima (Figura 1).

\section{Análise dos dados}

Análise de variância de duas vias (ANOVA two-way) foi realizada com o auxílio do programa Systat versão 10.0 (Wilkinson 2000) para determinar se havia diferenças entre a abundância de humanos, cães e aves nas áreas com alta e baixa concentração de humanos. Foram verificadas a normalidade dos resíduos e a homogeneidade de variâncias e quando alguma dessas premissas não foi satisfeita, a ANOVA foi substituída pelo teste não-paramétrico de Kruskal-Wallis. O teste de correlação de Spearman foi empregado com o auxílio do programa Bioestat versão 4.0 (Ayres et al. 2005) relacionando-se separadamente a abundância de cães e de humanos com a abundância de aves para cada área. Considerando que as coletas de dados foram realizadas em dias consecutivos, aumentando a probabilidade de contagem dos mesmos indivíduos, optou-se por empregar a média mensal de abundância de humanos, cães e aves para as análises descritas acima.

Para avaliar se havia diferenças na freqüência de registros de bandos e a quantidade de indivíduos por bando formados pelas aves entre as áreas, foram construídas tabelas de contingência testadas pelo Qui-Quadrado $\left(\chi^{2}\right)$ com fator de correção de Yates. Foi estabelecido o nível de significância $\alpha=5 \%$ para todas as análises.

\section{Resultados}

Foram efetuados 1966 registros de seis espécies de aves, Arenaria interpres (Linnaeus 1758, Scolopacidae) com 0,6\%, Calidris alba (Pallas 1764, Scolopacidae) com 3\%, Calidris canutus (Linnaeus 1758, Scolopacidae) com 0,2\%, Calidris pusilla (Linnaeus 1766, Scolopacidae) com 8,3\%, Charadrius semipalmatus (Bonaparte 1825, Charadriidae) com 85,7\% e Pluvialis dominica (Statius Muller 1776, Charadriidae) com 2,2\% dos registros. Somente Calidris canutus foi registrada exclusivamente na área de baixa concentração humana. $\mathrm{O}$ número médio e densidade total de aves, pessoas e cães encontramse na Tabela 1.

Houve diferença significativa no número médio mensal de pessoas (Teste de Kruskal-Wallis; $\mathrm{N}=6 ; \mathrm{H}=8,31 ; \mathrm{p}=0,004$ ) e número médio mensal de cães (ANOVA; $\mathrm{N}=6 ; \mathrm{F}=6,23 ; \mathrm{p}=0,05$ ) entre as áreas com alta e baixa concentração de humanos. No entanto, a diferença no número médio mensal de aves não foi significativa entre as áreas (ANOVA; $\mathrm{N}=6 ; \mathrm{F}=0,000 ; \mathrm{p}=0,985$ ) (Figura 2). As análises de correlação entre cães e humanos com aves não foram significativas nas duas áreas ( $\mathrm{p}>0,05)$ (Tabela 2).

Não houve diferença significativa na freqüência de registros de bandos $\left(\sum \chi^{2}=2,34 ; \mathrm{p}=0,126\right.$; Figura 3$)$ e no número médio de indivíduos por bando $\left(\sum \chi^{2}=0,05 ; \mathrm{p}=0,830\right.$; Figura 4$)$ das aves entre as áreas. A amplitude do número de aves por bando foi de 2 a 36 indivíduos na área com alta concentração e 2 a 62 indivíduos na área com baixa concentração humana.

\section{Discussão}

Os resultados do presente estudo rejeitam a hipótese de que a área com alta concentração humana, supostamente com maior risco de predação (Frid \& Dill 2002), interferiu negativamente na abundância das aves migratórias neárticas, pois a abundância geral das aves foi similar entre as áreas. Entretanto, esse resultado deve ser interpretado com cautela, pois aparentemente os limites máximos de concentração humana e de cães que as aves migratórias podem tolerar na área com alta concentração humana não foram atingidos no período do estudo. As aves provavelmente estejam enfrentando maiores condições de estresse provenientes de perturbações de pessoas e de cães na área com alta concentração humana, no entanto, é possível que essas condições ainda sejam insuficientes para afugentá-las do local (Yasué 2006, Burton 2007). Segundo Vooren \& Chiaradia (1990), a área de uso das aves costeiras torna-se inadequada quando a presença humana excede um determinado nível de perturbação. Dessa forma, a medida de tolerância das aves para perturbações resultantes da presença humana ainda é um fator a ser determinado em futuras pesquisas e, a densidade de pessoas e cães nas áreas de uso das aves deve estar relacionada primordialmente por representarem essas medidas das perturbações. No presente estudo, as densidades médias totais de $557 \pm 602$ pessoas $/ \mathrm{km}^{2}$ e $11 \pm 6$ cães $/ \mathrm{km}^{2}$ na área de alta concentração humana não foram suficientes para determinar padrões diferentes de abundância das aves entre as duas áreas.

A habituação à presença humana, a condição física das aves, os custos de forrageamento relacionados à qualidade e quantidade de hábitats disponíveis são fatores que também podem interferir na presença e abundância de aves migratórias em regiões impactadas pela

Tabela 1. Número médio total (+desvio padrão) e densidade de pessoas, cães domésticos e aves nas áreas com alta e baixa concentração humana amostradas entre novembro de 2006 a abril de 2007 em uma região de praia arenosa na costa sul do estado de São Paulo, Brasil.

Table 1. Total average (+standard deviation) and density of people, domestic dogs and birds of the areas with high and low human concentration on the south coast of São Paulo State, Brazil. Data surveyed from November 2006 to April 2007.

\begin{tabular}{lccccc}
\hline & \multicolumn{2}{c}{$\begin{array}{c}\text { Área com alta } \\
\text { concentração humana }\end{array}$} & & \multicolumn{2}{c}{$\begin{array}{c}\text { Área com baixa } \\
\text { concentração humana }\end{array}$} \\
\cline { 2 - 3 } \cline { 5 - 6 } & $\begin{array}{c}\text { Média } \\
(\mathbf{N}=\mathbf{6})\end{array}$ & $\begin{array}{c}\text { Densidade } \\
\left(\text { ind.km }^{-2}\right)\end{array}$ & & $\begin{array}{c}\text { Média } \\
(\mathbf{N}=\mathbf{6})\end{array}$ & $\begin{array}{c}\text { Densidade } \\
\left(\text { ind.km }^{-2}\right)\end{array}$ \\
\hline Pessoas & $223 \pm 241$ & $557 \pm 602$ & & $49 \pm 47$ & $123 \pm 118$ \\
Cães & $4 \pm 2$ & $11 \pm 6$ & & $2 \pm 2$ & $6 \pm 4$ \\
Aves & $8 \pm 4$ & $20 \pm 10$ & & $7 \pm 7$ & $17 \pm 17$ \\
\hline
\end{tabular}



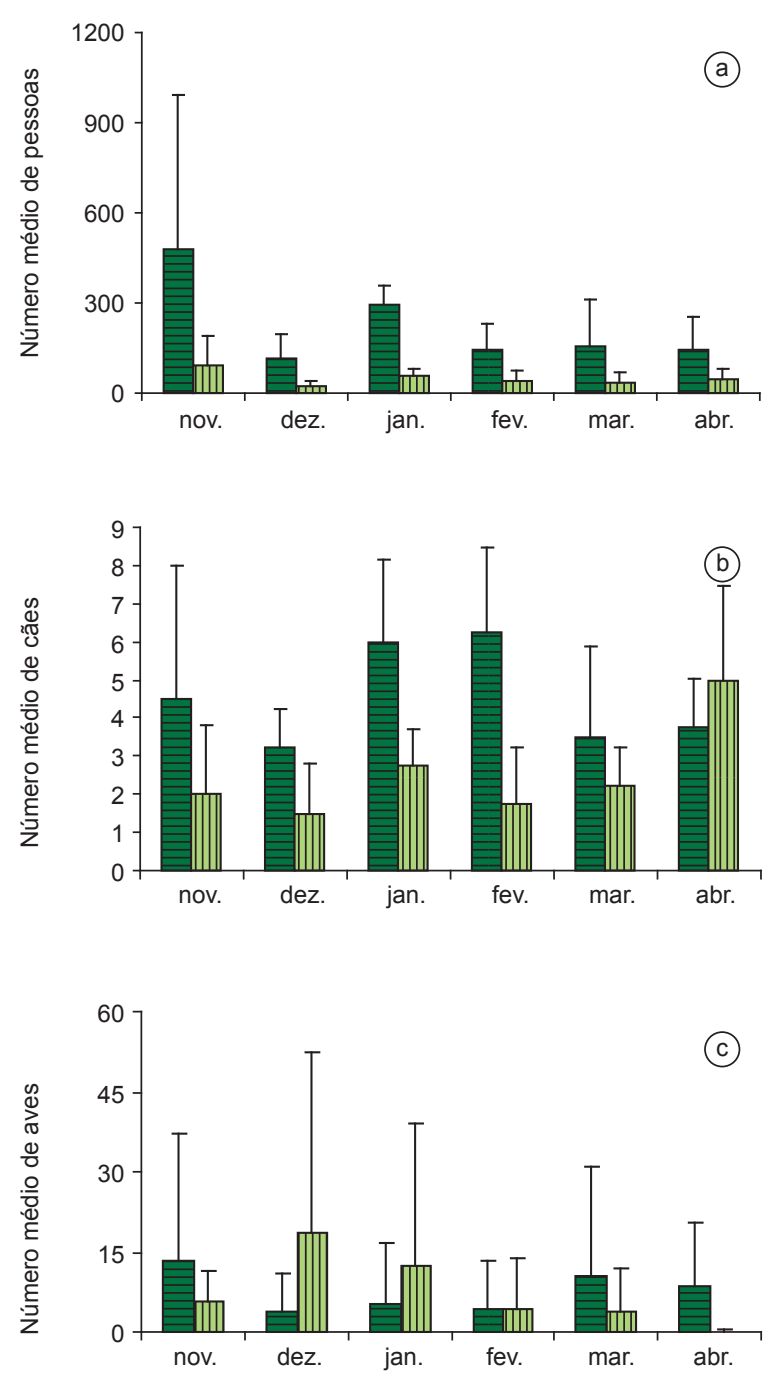

¿ Área com alta concentração humana

凹 Área com baixa concentração humana

Figura 2. Número médio mensal de pessoas a), cães b) e aves c) nas áreas com alta concentração e baixa concentração humana registrados entre novembro de 2006 a abril de 2007 em uma região de praia arenosa da costa sul do estado de São Paulo, Brasil. .

Figure 2. Monthly average of people a), dogs b) and birds c) in high and low human concentration areas surveyed from November 2006 to April 2007 on the south coast of São Paulo State, Brazil.

presença humana (Burger \& Gochfeld 1991, Nisbet 2000, Gill et al. 2001, Beale \& Monaghan 2004, Yasué 2006). Na maioria das ocasiões em que algum tipo de perturbação foi observado, como aproximação demasiada de pessoas e cães, as aves indicaram comportamentos de habituação, conforme descrito em Nisbet (2000), empreendendo vôos curtos e pousando alguns metros próximos ao ponto inicial de observação. Segundo Gill et al. (2001), esse tipo de comportamento também pode ser explicado pela mal condição física das aves, que respondem menos eficientemente às perturbações humanas; além disso, muitas vezes, a falta de hábitats com melhor qualidade e maior disponibilidade de alimentos também as restringem a permanecer, pelo menos temporariamente, em áreas com distúrbios. Em um estudo conduzido nas praias da região da baía de Delaware, um dos principais

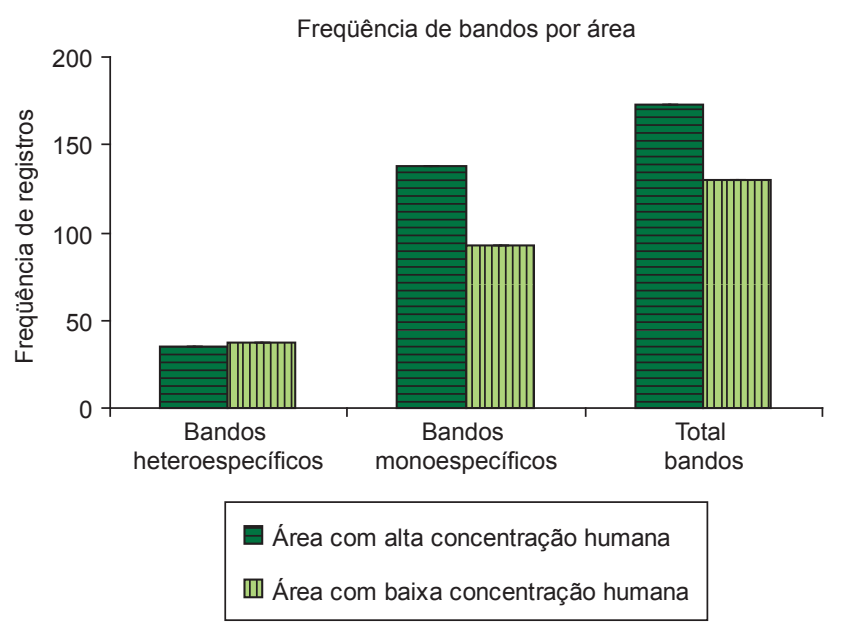

Figura 3. Frequiências de registros de bandos de aves entre as áreas com alta e baixa concentração humana entre novembro de 2006 a abril de 2007, em uma região de praia arenosa da costa sul do estado de São Paulo, Brasil.

Figure 3. Bird flocks frequencies of the high and low human concentration areas from November 2006 to April 2007 on the south coast of São Paulo State, Brazil.

Tabela 2. Coeficiente de correlação de Spearman para a relação de cães e humanos com aves nas áreas com alta e baixa concentração humana entre novembro de 2006 a abril de 2007 em uma região de praia arenosa da costa sul do estado de São Paulo, Brasil.

Table 2. Spearman coefficient results relating dogs and people with birds in the high and low human concentration areas from November 2006 to April 2007 on the south coast of São Paulo State, Brazil.

\begin{tabular}{lcccccc}
\hline & \multicolumn{2}{c}{$\begin{array}{c}\text { Área com alta } \\
\text { concentração } \\
\text { humana }\end{array}$} & & & \multicolumn{2}{c}{$\begin{array}{c}\text { Área com baixa } \\
\text { concentração } \\
\text { humana }\end{array}$} \\
\cline { 2 - 3 } \cline { 5 - 6 } & $\mathbf{r}_{\mathrm{s}}$ & $\mathbf{P}$ & & $\mathbf{r}_{\mathrm{s}}$ & $\mathbf{P}$ \\
\hline Cães $\mathrm{X}$ aves & 0,029 & 0,957 & & $-0,600$ & 0,208 \\
Humanos $\mathrm{x}$ aves & 0,714 & 0,111 & & $-0,086$ & 0,872 \\
\hline
\end{tabular}

$\mathrm{r}_{\mathrm{s}}$ coeficiente de correlação de Spearman.

pontos de pouso de aves migratórias limícolas na costa Atlântica dos Estados Unidos, Burger et al. (2004) relacionaram o comportamento de fuga e habituação das aves em resposta a perturbações humanas antes e após a tomada de medidas de proteção às aves. Com a tomada de medidas de proteção nas praias ao longo de 20 anos (instalação de placas com informações educativas, fiscalização, restrição ao acesso humano e de cães em áreas de uso freqüente das aves e construção de plataformas para observação das aves) a porcentagem de fuga das aves aumentou em relação ao período anterior. De acordo com os autores, após o estabelecimento dessas medidas, as aves encontraram praias com melhor qualidade para se movimentar quando perturbadas por atividades humanas, diminuindo os sinais de habituação à presença humana. No presente estudo, somente em ocasiões críticas de perturbações relacionadas à presença humana, como a perseguição contínua por cães domésticos, as aves se deslocaram por maiores distâncias, fora dos limites de observação. Thomas et al. (2003), sugerem que a presença de cães domésticos soltos pode ser um fator causador do decréscimo do número de aves mais significativo do que a própria perturbação humana em regiões costeiras. Segundo Burger et al. (2004), cães domésticos podem ser equivalentes a raposas e coiotes 


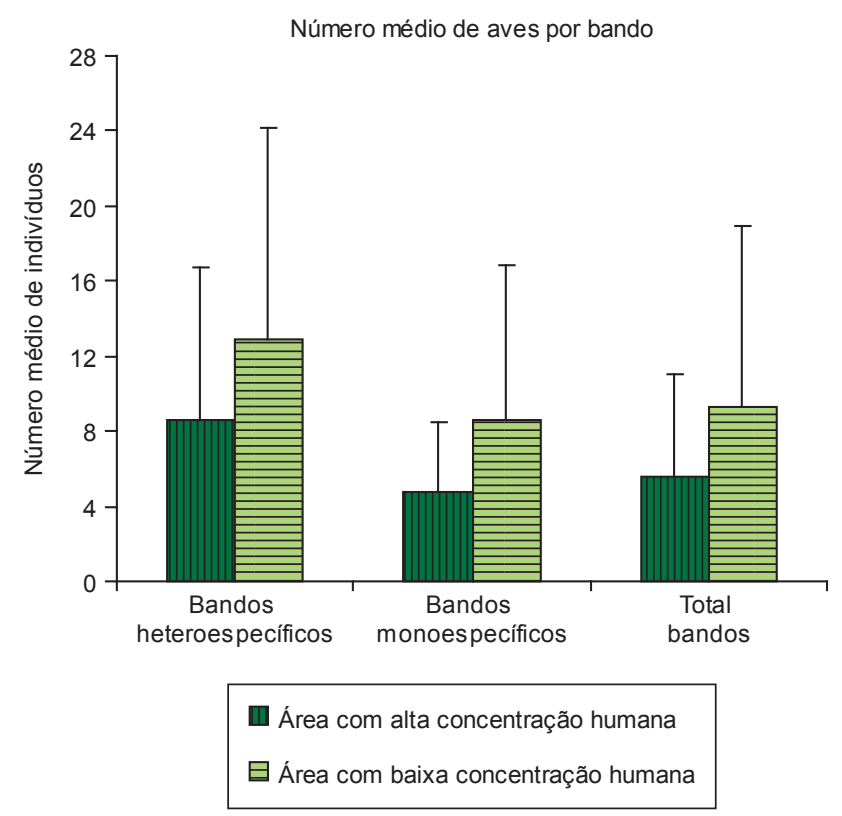

Figura 4. Número médio de aves por bando nas áreas com alta e baixa concentração humana registrado entre novembro de 2006 a abril de 2007 em uma região de praia arenosa da costa sul do estado de São Paulo, Brasil.

Figure 4. Average of the number of birds per flock of the high and low human concentration areas from November 2006 to April 2007 on the south coast of São Paulo State, Brazil.

de regiões temperadas, os quais são predadores freqüentes das aves migratórias.

Apesar do estabelecimento da área de transição de um quilômetro, na qual não foram coletados dados com o objetivo de discriminar claramente a presença de aves entre as áreas de alta e baixa concentração humana, é possível que o pouco isolamento da área de baixa concentração de locais com maiores concentrações de humanos tenha influenciado a inexistência significativa da variação da abundância de aves entre as áreas no presente estudo. Peters \& Otis (2007) indicam que estudos sobre respostas de indivíduos de aves em relação a perturbações humanas provavelmente possuem alta dependência com escalas geográficas adotadas. Assim, a relação de presença e abundância das aves migratórias neárticas com áreas de diferentes concentrações humanas poderia ser melhor representada em uma escala geográfica maior e com áreas mais isoladas da influência humana. Contudo, a falta de áreas nativas e não urbanizadas contínuas de extensões consideráveis paralelas às praias da região (Olmos \& Galetti 2004) provavelmente dificultarão o emprego de uma escala geográfica maior em futuros estudos sobre o tema.

A ausência de diferença significativa na frequiência de bandos e no número médio de indivíduos por bando entre as duas áreas amostradas não apóia a hipótese do maior efeito de proteção coletivo das aves na área com alta concentração humana (Elgard 1989, Cresswell 1994, Mori et al. 2001). Entretanto, em valores absolutos, a menor média, variabilidade e amplitude do número de indivíduos por bando na área com alta concentração humana provavelmente evidenciam aspectos relacionados à maior coesão dos indivíduos e à melhoria na eficiência de forrageamento das aves. De acordo com Lima \& Dill (1990) e Hilton et al. (1999), bandos mais coesos respondem mais rapidamente à ação de um predador, contudo podem despender um maior tempo vigilância para a proteção do grupo devido ao menor número de indivíduos sentinelas. Além disso, indivíduos freqüentadores de bandos menores evitam a redução local temporária de presas ao for- ragearem juntos, impedindo conseqüentemente a maior interferência comportamental negativa entre os membros (Yasué 2005).

Seis espécies de aves foram registradas dentre as 19 espécies de migratórias neárticas que podem utilizar as praias do estado de São Paulo (Figueiredo 2007). No entanto, Calidris canutus foi a única espécie registrada exclusivamente na área de baixa concentração humana, o que indica que é sensível a condições com maior concentração e perturbação humana, tal como observado por Peters e Otis (2007) em um estudo conduzido na Carolina do Sul (EUA). Estes autores sugeriram que a espécie evita locais de refúgios de aves presentes em distâncias menores de $1000 \mathrm{~m}$ de áreas com alta atividade de barcos utilizados para pesca esportiva. Apesar de sua distribuição incluir toda a costa brasileira durante o período de invernada (Sick 1997), C. canutus é pouco freqüente e em alguns estudos não possui sequer registros (Telino-Júnior et al. 2003, Branco et al. 2004, Cabral et al. 2006), ao contrário de outras espécies pertencentes ao mesmo gênero, como C. alba e C. pusilla, que normalmente são mais abundantes ao longo da costa brasileira (Rodrigues 2000).

A população de $C$. canutus rufa entrou em declínio durante os últimos anos tornando-se motivo de preocupação para sua conservação (Vooren \& Brusque 1999, Wetlands International 2002, Morrison et al. 2004). Segundo Morrison et al. (2004), esta espécie apresenta características que a torna atualmente vulnerável, tais como tendência de uso de um número limitado de locais durante a migração, baixa fecundidade, migração sincronizada com a abundância de suas principais presas e alimentos (p. ex. ovos do caranguejo Limulus polyphemus na baía de Delaware, EUA) e ocupação de áreas que estão sendo gradativamente invadidas por humanos.

As aves migratórias costeiras necessitam de locais com condições ambientais adequadas para sobreviver (Vooren \& Brusque 1999). Nesse sentido, considerando a escassez de estudos sobre as conseqüências da influência humana para as aves migratórias costeiras no Brasil, é recomendável o estabelecimento dos limites de perturbação humana que as aves podem tolerar em futuras pesquisas para a tomada de medidas de proteção, principalmente durante o período de invernagem no qual costumam aparecer em maiores quantidades na costa do Brasil.

\section{Agradecimentos}

Meus sinceros agradecimentos a dois revisores anônimos que contribuíram com sugestões presentes neste manuscrito.

\section{Referências Bibliográficas}

AZEVEDO-JÚNIOR, S.M., DIAS, M.M., LARRAZÁBAL, M.E., JÚNIOR, W.R.T., LYRA-NEVES, R.M. \& FERNANDES, C.J.G. 2001. Recapturas e recuperações de aves migratórias no litoral de Pernambuco. Ararajuba 9(1):33-42.

AYRES, M.M., AYRES, JR., AYRES, D.L. \& SANTOS, A.S. 2005. BioEstat 4.0: Aplicações estatísticas nas áreas das ciências biológicas e médicas. Sociedade Civil Mamirauá/MCT-CNPq/Conservation International, Belém, 323 p.

BEALE, C.M. \& MONAGHAN, P. 2004. Behavioral responses to human disturbance a matter of choice? Anim. Behav. 68:1065-1069.

BRANCO, J.O., MACHADO, I.F. \& BOVENDORP, M.S. 2004. Avifauna associada a ambientes de influência marítima no litoral de Santa Catarina, Brasil. Rev. Bras. Zool. 21(3):459-466.

BURGER, J. 1981. The effect of human activity on birds at a coastal bay. Biol. Cons. 21:231-241.

BURGER, J. \& GOCHFELD, M. 1991. Human activity influence and diurnal and nocturnal foraging of Sanderlings. Condor 93:259-265.

BURGER, J. 1993. Shorebird squeeze. Nat. Hist. 102:8-12. 
BURGER, J., JEITNER, C., CLARK, K. \& NILES, J.L. 2004. The effect of human activities on migrant shorebirds:successful adaptive management. Environ. Conserv. 31(4):283-288.

BURTON, N.H.K., REHFISCH, M.M., CLARK, N.A. \& DODD, S.G. 2006. Impacts of sudden winter habitat loss on the body condition and survival of Redshank Tringa totanus. J. Appl. Ecol. 43:464-473.

BURTON, N.H.K. 2007. Landscape approaches to studying the effects of disturbance on waterbirds. Ibis 49:95-101.

CABRAL, S.A.S., AZEVEDO-JÚNIOR, S.M. \& LARRAZÁBAL, M.E. 2006. Abundância sazonal de aves migratórias na Área de Proteção Integral de Piaçabuçu, Alagoas, Brasil. Rev. Bras. Zool. 23(3):865-869.

CRESSWELL, M.A. 1994. Flocking as an effective anti-predation strategy in redshanks, Tringa totanus. Anim. Behav. 47:433-442.

ELGARD, M.A. 1989. Predator vigilance and group size in mammals and birds: a critical review of the empirical evidence. Biol. Rev. 64:13-23.

FIGUEIREDO, L.F.A. 2007. Lista das aves do Estado de São Paulo. http://www.ib.usp.br/ceo. (último acesso em 02/10/2007).

FRID, A. \& DILL, L. 2002. Human-caused disturbance stimuli as a form of predation risk. Conserv. Ecol. 6:11-26.

GILL, J.A., NORRIS, K. \& SUTHERLAND, W.J. 2001. Why behavioral responses may not reflect the population consequences of human disturbance. Biol. Conserv. 97:265-268.

HILTON, G.M., CRESSWELL, W. \& HUXTON, G.D. 1999. Intraflock variation in the speed of escape-flight response on attack by an avian predator. Behav. Ecol. 10(4):391-395.

HUBBARD, D.M. \& DUGAN, J.E. 2003. Shorebird use of an exposed sandy beach in southern California. Estuar. Cost. Shelf Science 58:41-54.

INSTITUTO BRASILEIRO DE GEOGRAFIA E ESTATÍSTICA (IBGE). 2000. Censo Demográfico. http://www.ibge.gov.br (último acesso em 12/08/2007).

LARRAZÁBAL, M.E., AZEVEDO-JÚNIOR, S.M. \& PENA, O. 2002. Monitoramento de aves limícolas na salina Diamante Branco, Galinhos, Rio Grande do Norte, Brasil. Rev. Bras. Zool. 19(4):1081-1089.

LIMA, S.L. \& DILL, L.M. 1990. Behavioural decisions made under the risk of predation: a review and prospectus. Can. J. Zool. 68:619-640.

MORI, Y., SODHI N.S., KAWANISHI, S. \& YAMAGISHI, S. 2001. The effect of human disturbance and flock composition on the flight distance of waterfowl species. J. Ethol. 19:115-119.

MORRISON, R.I.G., ROSS, R.K. \& ZANTAS, P.T. 1989. Brazil In Atlas of Nearctic shorebirds on the coast of South America (Morrison, R.I.G. \& Ross, R.K. eds.). Canadian Wildlife Service, Ottawa, p. 179-211.
MORRISON, R.I.G., ROSS, R.K. \& NILES, L.M. 2004. Declines in wintering populations of Red Knots in Southern South America. Condor 106:60-70.

NISBET, I.C.T. 2000. Disturbance, habituation, and management of waterbird colonies. Waterbirds 23:312-332.

OLMOS, F. \& GALETTI, M. 2004. A conservação e o futuro da Juréia: isolamento ecológico e impacto humano. In Estação Ecológica JuréiaItatins: Ambiente Físico, Flora e Fauna. (Marques, O.A.V. \& Duleba, W. eds.). Holos, Ribeirão Preto, p. 360-377.

PETERS, K.A. \& OTIS, D.L. 2007. Shorebirds roost-site selection at two temporal scales: is human disturbance a factor? J. Appl. Ecol. 44:196-209.

RODRIGUES, A.A.F. 2000. Seasonal abundance of Nearctic shorebirds in the Gulf of Maranhão. J. Field Ornithol. 71(4):665-675.

SICK, H. 1997. Ornitologia Brasileira. Nova Fronteira, Rio de Janeiro, $912 \mathrm{p}$.

TELINO-JÚNIOR, W.R., AZEVEDO-JÚNIOR, S.M. \& LYRA-NEVES, R.M. 2003. Censo de aves migratórias na Coroa do Avião, Igarassu, Pernambuco, Brasil. Rev. Bras. Zool. 20(3):451-456.

THOMAS, K., KVITEK, R.G. \& BRETZ, C. 2003. Effects of human activity on the foraging behavior of sanderlings Calidris alba. Biol. Conserv. 109:67-71.

VOOREN, C. M. \& CHIARADIA, A. 1990. Seasonal abundance and behavior of coastal birds on Cassino beach, Brazil. Ornitol. Neotrop. 1:9-24.

VOOREN, C.M. \& BRUSQUE, L. 1999. Diagnóstico sobre as aves do ambiente costeiro do Brasil. Avaliação e ações prioritárias para conservação da biodiversidade da zona costeira e marinha Pronabio. http://www.anp.gov.br. (ultimo acesso em 15/08/2008).

WETLANDS INTERNATIONAL. 2002. Waterbird population estimates. $3^{\text {rd }}$ ed. Wetlands International Global Series, No. 12. Wetlands International, Wageningen.

WILKINSON, L. 2000. Systat: the system for statistics. Evanston, Illinois.

YASUÉ, M. 2005. The effects of human presence, flock size and prey density on shorebird foraging rates. J. Ethol. 23:199-204.

YASUÉ, M. 2006. Environmental factors and spatial scale influence shorebirds' responses to human disturbance. Biol. Conserv. 128:47-54.

Recebido em 01/06/08 Versão reformulada recebida em 25/10/08 Publicado em 06/11/08 